\title{
On Projective Reconstruction In Arbitrary Dimensions
}

\author{
Behrooz Nasihatkon, Richard Hartley \\ Australian National University \\ National ICT Australia (NICTA)* \\ behrooz.nasihatkon@anu.edu.au, richard.hartley@anu.edu.au
}

\author{
Jochen Trumpf \\ Australian National University \\ Jochen. Trumpf@anu . edu . au
}

\begin{abstract}
We study the theory of projective reconstruction for multiple projections from an arbitrary dimensional projective space into lower-dimensional spaces. This problem is important due to its applications in the analysis of dynamical scenes. The current theory, due to Hartley and Schaffalitzky, is based on the Grassmann tensor, generalizing the ideas of fundamental matrix, trifocal tensor and quadrifocal tensor used in the wellstudied case of $3 D$ to $2 D$ projections. We present a theory whose point of departure is the projective equations rather than the Grassmann tensor. This is a better fit for the analysis of approaches such as bundle adjustment and projective factorization which seek to directly solve the projective equations. In a first step, we prove that there is a unique Grassmann tensor corresponding to each set of image points, a question that remained open in the work of Hartley and Schaffalitzky. Then, we prove that projective equivalence follows from the set of projective equations given certain conditions on the estimated camera-point setup or the estimated projective depths. Finally, we demonstrate how wrong solutions to the projective factorization problem can happen, and classify such degenerate solutions based on the zero patterns in the estimated depth matrix.
\end{abstract}

\section{Introduction}

This paper contains new theoretical results on projective reconstruction for multiple projections from an arbitrary dimensional projective space $\mathbb{P}^{r-1}$ to lowerdimensional spaces $\mathbb{P}^{s_{i}-1}$. A set of such projections can be represented as

$$
\lambda_{i j} \mathbf{x}_{i j}=\mathrm{P}_{i} \mathbf{X}_{j}
$$

*NICTA is funded by the Australian Government as represented by the Department of Broadband, Communications and the Digital Economy and the Australian Research Council through the ICT Centre of Excellence program. where $\mathbf{X}_{j} \in \mathbb{R}^{r}$ is the $j$-th high-dimensional (HD) point, $\mathrm{P}_{i} \in \mathbb{R}^{s_{i} \times r}$ is the $i$-th projection matrix, representing a projection $\mathbb{P}^{r-1} \rightarrow \mathbb{P}^{s_{i}-1}, \mathbf{x}_{i j} \in \mathbb{R}^{s_{i}}$ is an image point and $\lambda_{i j}$-s are nonzero scalars known as projective depths. Projective reconstruction is to obtain the projection matrices $\mathrm{P}_{i}$, the points $\mathbf{X}_{j}$ and the depths $\lambda_{i j}$, up to a projective ambiguity, given the image points $\mathbf{x}_{i j}$. This paper extends the current reconstruction theory, which is mostly fit for the tensorbased approaches, and provides theoretical tools for the analysis of the reconstruction methods, like projective factorization, in which the set of equations (1) is directly solved. We also fully specify possible incorrect solutions obtained by projective factorization methods.

The classic case of projections from $3 \mathrm{D}$ scenes to $2 \mathrm{D}$ images $\left(r=4, s_{i}=3\right)$ has been intensely studied in the past two decades [4]. When the scene is rigid, the traditional way of analysing and solving the problem of projective reconstruction is via the bifocal tensor (fundamental matrix), trifocal tensor or quadrifocal tensor $[4,5]$. The standard procedure is to build a multi-view tensor from point (or line) correspondences, extract the camera matrices, up to projectivity, from the tensor, and estimated the 3D points through triangulation.

The recovery of structure and motion is more challenging in the case of nonrigid motions. Wolf and Shashua [12] consider several structure and motion problems in which the scene observed by a perspective camera is nonrigid. They show that all those problems can be modeled as projections from a higherdimensional projective space $\mathbb{P}^{k}$ into $\mathbb{P}^{2}$ for $k=$ $3,4,5,6$. They use tensorial approaches to address each of the problems. Hartley and Vidal [2] consider the problem of perspective nonrigid deformation, assuming that the scene deforms as linear combination of $k$ linearly independent basis shapes. They show that the problem can be modeled as projections from $\mathbb{P}^{3 k}$ to $\mathbb{P}^{2}$.

Such applications manifest the need for a general theory of projective reconstruction for arbitrary dimensional spaces. Hartley and Schaffalitzky [3] present 
such a theory by introducing the Grassmann tensor, generalizing the concepts of bifocal, trifocal and quadrifocal tensors used for $\mathbb{P}^{3} \rightarrow \mathbb{P}^{2}$ projections, and other tensors which had been introduced for special cases in other dimensions. The central theorem in [3] proves that the projection matrices can be obtained up to projectivity from the corresponding Grassmann tensor.

Tensor-based projective reconstruction is sometimes not accurate enough, especially in the presence of noise. One problem is imposing certain nonlinear constraints on the tensor. While the tensor is determined using the linear constraints imposed by point correspondences, one should also consider the internal constraints which are intrinsic to each type of multi-view tensor. For instance, the fundamental matrix (bifocal tensor) is known to have a zero determinant which imposes a single polynomial constraint. This is the only required constraint. The number of such internal constraints grows dramatically with the dimensionality of the multi-view tensor. For example, the trifocal tensor is known to have 8 internal constraints. For the quadrifocal tensor this number is 51 (see [4, Sect. 17.5]). The number of internal constraints is even larger for projections from higher dimensions. As the tensors are usually estimated linearly, imposing such constraints can be an issue specially when data is noisy.

Another issue is that a multi-view tensor can be defined only for up to a limited number of views. This limit is four views for $\mathbb{P}^{3} \rightarrow \mathbb{P}^{2}$ projections [4]. In general, for projections from $\mathbb{P}^{r-1}$, at most $r$ views can be involved in multilinear relations corresponding to a single tensor [3]. This prevents us from using data from all views to reduce the estimation error. Such issues has led to the use of other approaches such as bundle adjustment [11] and projective factorization $[10,6,9,1]$ in which the projection equations (1) are directly solved for projection matrices $\mathrm{P}_{i}$, points $\mathbf{X}_{j}$ and depths $\lambda_{i j}$. Such approaches are either used in conjunction with a tensor method or independently. Analysing such methods requires a theory which derives projective reconstruction from the projection equations (1), rather than the multi-view tensor. Providing such a theory is the purpose of this paper.

As a first step, we need to answer a question which is left open in [3], namely whether sufficiently many image points $\mathbf{x}_{i j}$ uniquely determine the multi-view (Grassmann) tensor. Notice that this is important even for tensor-based reconstruction. The uniqueness of the multi-view tensor has been shown in section 3 .

The second question is when an estimated configuration of projections and points satisfying relations (1) is projectively equivalent to the true configuration. This is important for the analysis of bundle adjustment as well as projective factorization. The answer to such a simple question is by no means trivial. Notice that the uniqueness of the Grassmann tensor is not sufficient for answering this, as it does not rule out the existence of degenerate solutions $\left\{\mathrm{P}_{i}\right\}$ for which the corresponding Grassmann tensor is zero. In sections 4 and 5 we respectively give conditions on the estimated projectionpoint setup and the estimated projective depths under which projective equivalence holds. Especially, in Sect. 5 we show that the two setups are projectively equivalent if the estimated projective depths are all nonzero.

The last issue, which only concerns the projective factorization approaches, is classifying the wrong solutions to the projective factorization equation

$$
\Lambda \odot\left[\mathbf{x}_{i j}\right]=\mathrm{PX} .
$$

This is the matrix form of (1) and shows the idea behind factorization-based methods: find an estimation of the depth matrix $\Lambda=\left[\lambda_{i j}\right]$ such that the matrix of weighted image points $\Lambda \odot\left[\mathbf{x}_{i j}\right]=\left[\lambda_{i j} \mathbf{x}_{i j}\right]$ has a rank- $r$ factorization PX. While the true projective depths are naturally nonzero, it is difficult or inefficient to directly constrain all the depths estimated by such algorithms away from zero. It is known that the iterative factorization algorithms may give degenerate solutions in which a subset of projective depths converge to zero, as shown by Oliensis and Hartley [9]. This is why almost all such algorithms restrict or balance the depth matrix during estimation. We refer the readers to [8] for a review such algorithms and the constraints used.

The constraints are mostly aimed to avoid zero rows and zero columns in the depth matrix, which are the two well-known classes of degenerate solutions. Recent work by the authors [8] shows that, for $\mathbb{P}^{3} \rightarrow \mathbb{P}^{2}$ projections, apart from the solutions with zero rows or zero columns, there exists one and only one class of wrong solutions in which the depth matrix has a cross-shaped structure. In Sect. 6 we extend this result by giving a classification of the wrong solutions for arbitrary dimensional projections based on the zero patterns in the depth matrix. It turns out that the form of such degenerate solutions varies from case to case, and in general, can be much more complex compared to $3 \mathrm{D}$ to $2 \mathrm{D}$ projections. Besides verification of the solutions given by an algorithm, the importance of this result is that the constraints on projective depths need only rule out specific classes of zero patterns rather than preventing all projective depths from converging to zero.

Due to space constraints, we cannot provide the full proofs here. The object of this paper is, therefore, to list the main results and give the general outline of the proofs. We refer the reader to [7] for full proofs and more details. 


\section{Background}

\subsection{Conventions}

We use typewriter letters (A) for matrices, bold letters $(\mathbf{a}, \mathbf{A})$ for vectors, lower-case normal letters $(a)$ for scalars and upper-case normal letters $(A)$ for sets, except for special sets like $\mathbb{R}$ and $\mathbb{P}$. Calligraphic letters $(\mathcal{A})$ are used for both tensors and mappings (functions). To refer to the column space and null space of a matrix $\mathrm{A}$ we respectively use $\mathcal{C}(\mathrm{A})$ and $\mathcal{N}(\mathrm{A})$. The vertical concatenation of a set of matrices $A_{1}, A_{2}, \ldots, A_{m}$ is denoted by $\operatorname{stack}\left(A_{1}, \ldots, A_{m}\right)$. We make use of the terms "generic" and "in general position" for entities such as points, matrices and subspaces. In such cases, if the generic properties are not explicitly stated, we mean that those objects belong to some open and dense subset which is implicitly determined from the proofs.

Throughout the paper we deal with a true setup $\left(\left\{\mathrm{P}_{i}\right\},\left\{\mathbf{X}_{j}\right\},\left\{\lambda_{i j}\right\}\right)$ and an estimated setup $\left(\left\{\hat{\mathbf{P}}_{i}\right\},\left\{\hat{\mathbf{X}}_{j}\right\},\left\{\hat{\lambda}_{i j}\right\}\right)$. The true setup is the actual configuration of projections, HD points and depths from which the image points are created as $\mathbf{x}_{i j}=\mathrm{P}_{i} \mathbf{X}_{j} / \lambda_{i j}$. The estimated setup, denoted by hatted quantities, is obtained from the image points $\mathbf{x}_{i j}$, supposedly by a reconstruction algorithm, such that it satisfies all the projection equations $\hat{\lambda}_{i j} \mathbf{x}_{i j}=\hat{\mathrm{P}}_{i} \hat{\mathbf{X}}_{j}$. One can usually make certain genericity assumptions about the true projection-point setup $\left(\left\{\mathrm{P}_{i}\right\},\left\{\mathbf{X}_{j}\right\}\right)$ or safely assume $\lambda_{i j} \neq 0$ for all $i, j$. Such natural assumptions cannot be as easily made about the estimated quantities, as they are to be obtained by an algorithm. It is our goal to present minimal conditions on the estimated $\operatorname{setup}\left(\left\{\hat{\mathrm{P}}_{i}\right\},\left\{\hat{\mathbf{X}}_{j}\right\},\left\{\hat{\lambda}_{i j}\right\}\right)$ under which $\left(\left\{\mathrm{P}_{i}\right\},\left\{\mathbf{X}_{j}\right\}\right)$ and $\left(\left\{\hat{\mathrm{P}}_{i}\right\},\left\{\hat{\mathbf{X}}_{j}\right\}\right)$ are projectively equivalent. We usually combine the two equations $\lambda_{i j} \mathbf{x}_{i j}=\mathrm{P}_{i} \mathbf{X}_{j}$ and $\hat{\lambda}_{i j} \mathbf{x}_{i j}=$ $\hat{\mathrm{P}}_{i} \hat{\mathbf{X}}_{j}$ after a simple change of variables $\mathbf{x}_{i j} \leftarrow \lambda_{i j} \mathbf{x}_{i j}$, $\hat{\lambda}_{i j} \leftarrow \hat{\lambda}_{i j} / \lambda_{i j}$ to get $\hat{\mathrm{P}}_{i} \hat{\mathbf{X}}_{j}=\hat{\lambda}_{i j} \mathrm{P}_{i} \mathbf{X}_{j}$. This change of variables does not affect our results as there are no assumptions about $\mathbf{x}_{i j}$-s and the only assumption about a projective depth $\hat{\lambda}_{i j}$ is whether or not it is zero.

We must stress that, here, the projection matrices $\mathrm{P}_{i}, \hat{\mathrm{P}}_{i}$, HD points $\mathbf{X}_{j}, \hat{\mathbf{X}}_{j}$ and image points $\mathbf{x}_{i j}$ are treated as members of a real vector space, even though they might represent quantities in a projective space. For instance, $\mathbf{X}_{j} \in \mathbb{R}^{r}$ represents a point in $\mathbb{P}^{r-1}$ in homogeneous coordinates. Any equality sign "=" here is strict and never implies equality up to scale.

\subsection{Projective equivalence}

We formalize the concept of projective equivalence for HD points and projection matrices as follows.
Definition 1. Two sets of projection matrices $\left\{\mathrm{P}_{i}\right\}$ and $\left\{\hat{\mathrm{P}}_{i}\right\}$, with $\mathrm{P}_{i}, \hat{\mathrm{P}}_{i} \in \mathbf{R}^{s_{i} \times r}$ for $i=1,2, \ldots, m$ are projectively equivalent if there exist nonzero scalars $\tau_{1}, \tau_{2}, \ldots, \tau_{m}$ and an $r \times r$ invertible matrix $\mathrm{H}$ such that

$$
\hat{\mathrm{P}}_{i}=\tau_{i} \mathrm{P}_{i} \mathrm{H}, \quad i=1,2, \ldots, m .
$$

Two sets of points $\left\{\mathbf{X}_{j}\right\}$ and $\left\{\hat{\mathbf{X}}_{j}\right\}$ with $\mathbf{X}_{j}, \hat{\mathbf{X}}_{j} \in \mathbb{R}^{r}$ for $j=1,2, \ldots, n$, are projectively equivalent if there exist nonzero scalars $\nu_{1}, \nu_{2}, \ldots, \nu_{n}$ and an invertible $r \times r$ matrix $\mathrm{G}$ such that

$$
\hat{\mathbf{X}}_{j}=\nu_{j} \mathrm{G} \mathbf{X}_{j}, \quad j=1,2, \ldots, n .
$$

Two setups $\left(\left\{\mathrm{P}_{i}\right\},\left\{\mathbf{X}_{j}\right\}\right)$ and $\left(\left\{\hat{\mathrm{P}}_{i}\right\},\left\{\hat{\mathbf{X}}_{j}\right\}\right)$ are projectively equivalent if (3) and (4) hold with $\mathrm{G}=\mathrm{H}^{-1}$.

Lemma 1. Consider a set of points $\mathbf{X}_{1}, \mathbf{X}_{2}, \ldots, \mathbf{X}_{n} \in$ $\mathbb{R}^{r}$ with $n>r$ with the generic properties

(P1) $\operatorname{span}\left(\mathbf{X}_{1}, \ldots, \mathbf{X}_{n}\right)=\mathbb{R}^{r}$, and

(P2) the set of points $\left\{\mathbf{X}_{i}\right\}$ cannot be partitioned into $p \geq 2$ nonempty subsets, such that subspaces defined as the span of each subset are independent ${ }^{1}$.

Now, for any set of points $\left\{\hat{\mathbf{X}}_{i}\right\}$ projectively equivalent to $\left\{\mathbf{X}_{i}\right\}$, the matrix $\mathrm{G}$ and scalars $\nu_{j}$ defined in (4) are unique up to a scale ambiguity of the form $\left(\beta \mathrm{G},\left\{\nu_{j} / \beta\right\}\right)$ for any nonzero scalar $\beta$.

Notice that (P2) is generic only when $n>r$. The proof [7] is based on the theory of eigenspaces.

\subsection{Triangulation}

The problem of Triangulation is to find a point $\mathbf{X}$ given its images through a set of known projections $\mathrm{P}_{1}, \ldots, \mathrm{P}_{m}$. The next lemma provides conditions for the uniqueness of triangulation.

Lemma 2 (Triangulation). Consider a set of projection matrices $\mathrm{P}_{1}, \mathrm{P}_{2}, \ldots, \mathrm{P}_{m}$ with $\mathrm{P}_{i} \in \mathbb{R}^{s_{i} \times r}$, and a point $\mathbf{X} \in \mathbb{R}^{r}$, configured such that

(T1) there does not exist any linear subspace of dimension one or two, passing through $\mathbf{X}$ and nontrivially intersecting ${ }^{2}$ all the null spaces $\mathcal{N}\left(\mathrm{P}_{1}\right), \mathcal{N}\left(\mathrm{P}_{2}\right), \ldots, \mathcal{N}\left(\mathrm{P}_{m}\right)$.

Now, for any nonzero $\mathbf{Y} \neq \mathbf{0}$ in $\mathbb{R}^{r}$ if the relations

$$
\mathrm{P}_{i} \mathbf{Y}=\beta_{i} \mathrm{P}_{i} \mathbf{X}, \quad i=1,2, \ldots, m
$$

hold for scalars $\beta_{i}$, then $\mathbf{Y}=\beta \mathbf{X}$ for some scalar $\beta \neq 0$.

\footnotetext{
${ }^{1}$ Subspaces $U_{1}, \ldots, U_{p}$ are independent if $\operatorname{dim}\left(\sum_{j=1}^{p} U_{j}\right)=$ $\sum_{j=1}^{p} \operatorname{dim}\left(U_{j}\right)$, where $\sum_{j=1}^{p} U_{j}=\left\{\sum_{j=1}^{p} \mathbf{u}_{j} \mid \mathbf{u}_{j} \in U_{j}\right\}$.

${ }^{2}$ Two linear subspaces nontrivially intersect if their intersection has dimension one or more.
} 
Notice that we have not assumed $\beta_{i} \neq 0$. For the classic case of projections $\mathbb{P}^{3} \rightarrow \mathbb{P}^{2}$, (T1) simply means that the camera centres $\mathcal{N}\left(\mathrm{P}_{i}\right) \in \mathbb{P}^{3}$ and the projective point $\operatorname{span}(\mathbf{X}) \in \mathbb{P}^{3}$ are not (projectively) collinear. Besides the proof, in [7] we show that (T1) is a generic property for arbitrary dimensional projections.

\subsection{Valid profiles and the Grassmann tensor}

Consider a set of projection matrices $\mathrm{P}_{1}, \mathrm{P}_{2}, \ldots, \mathrm{P}_{m}$, with $\mathrm{P}_{i} \in \mathbb{R}^{s_{i} \times r}$, such that $\sum_{i=1}^{m}\left(s_{i}-1\right) \geq r$. A valid profile [3] is defined as an $m$-tuple of nonnegative ${ }^{3}$ integers $\boldsymbol{\alpha}=\left(\alpha_{1}, \alpha_{2}, \ldots, \alpha_{m}\right)$ such that $0 \leq \alpha_{i} \leq s_{i}-1$ and $\sum \alpha_{i}=r$. Clearly, there might exist different valid profiles for a setup $\left\{\mathrm{P}_{i}\right\}$. One can choose $r \times r$ submatrices of $\mathrm{P}=\operatorname{stack}\left(\mathrm{P}_{1}, \mathrm{P}_{2}, \ldots, \mathrm{P}_{m}\right)$ according to a profile $\boldsymbol{\alpha}$, by choosing $\alpha_{i}$ rows from each $\mathrm{P}_{i}$. Notice that due to the property $\alpha_{i} \leq s_{i}-1$, all the rows of $\mathrm{P}_{i}$ are never chosen for building such a submatrix.

Consider $m$ index sets $I_{1}, I_{2}, \ldots, I_{m}$, such that each $I_{i}$ contains the indices of some $\alpha_{i}$ rows of $\mathrm{P}_{i}$. Each way of choosing $I_{1}, I_{2}, \ldots, I_{m}$ gives a square submatrix of $\mathrm{P}=\operatorname{stack}\left(\mathrm{P}_{1}, \ldots, \mathrm{P}_{m}\right)$ where the rows of each $\mathrm{P}_{i}$ are chosen in order. The determinant of this submatrix is multiplied by a corresponding sign ${ }^{4}$ to form $\mathcal{T}_{\boldsymbol{\alpha}}^{I_{1}, I_{2}, \ldots, I_{m}}$, which is a Grassmann coordinate of $\mathcal{C}(\mathrm{P})$, the column space of $\mathrm{P}$. Such entries for different choices of the $I_{i}$-s can be arranged in a multidimensional array $\mathcal{T}_{\boldsymbol{\alpha}}$ called the Grassmann tensor corresponding to $\boldsymbol{\alpha}$. The dimension of $\mathcal{T}_{\boldsymbol{\alpha}}$ is equal to the number of nonzero entries of $\boldsymbol{\alpha}=\left(\alpha_{1}, \alpha_{2}, \ldots, \alpha_{m}\right)$, as $\mathcal{T}_{\boldsymbol{\alpha}}$ does not depend on those $\mathrm{P}_{i}$ with $\alpha_{i}=0$. To show the dependence of the Grassmann tensor on projection matrices $\mathrm{P}_{i}$, we sometimes use the mapping $\mathcal{G}_{\boldsymbol{\alpha}}$ which takes a set of projection matrices to the corresponding Grassmann tensor, that is $\mathcal{T}_{\boldsymbol{\alpha}}=\mathcal{G}_{\boldsymbol{\alpha}}\left(\mathrm{P}_{1}, \mathrm{P}_{2}, \ldots, \mathrm{P}_{m}\right)$. Notice that $\mathcal{G}_{\boldsymbol{\alpha}}$ itself is not a tensor. Obviously, $\mathcal{G}_{\boldsymbol{\alpha}}\left(\mathrm{P}_{1}, \ldots, \mathrm{P}_{m}\right)$ is nonzero if and only if $\mathrm{P}$ has at least one non-singular submatrix chosen according to $\boldsymbol{\alpha}$.

Hartley and Schaffalitzky [3] show that if a point $\mathbf{X}$ is projected through $\mathrm{P}_{1}, \mathrm{P}_{2}, \ldots, \mathrm{P}_{m}$ into the image points $\mathbf{x}_{1}, \mathbf{x}_{2}, \ldots, \mathbf{x}_{n}$, according to $\lambda_{i} \mathbf{x}_{i}=\mathrm{P}_{i} \mathbf{X}$, then for any set of full-column-rank matrices $\mathrm{U}_{1}, \mathrm{U}_{2}, \ldots, \mathrm{U}_{m}$ with $\mathrm{U}_{i} \in \mathbb{R}^{s_{i} \times\left(s_{i}-\alpha_{i}\right)}$ and $\mathbf{x}_{i} \in \mathcal{C}\left(\mathrm{U}_{i}\right)$ we have

$$
\sum_{I_{1}, \ldots, I_{m}} \mathcal{T}_{\boldsymbol{\alpha}}^{I_{1}, I_{2}, \ldots, I_{m}} \prod_{i=1}^{m} \operatorname{det}\left(\mathrm{U}_{i}^{\bar{I}_{i}}\right)=0
$$

\footnotetext{
${ }^{3}$ Notice that, the definition of a valid profile here slightly differs from [3] which needs $\alpha_{i} \geq 1$. We choose this new definition for convenience, as it does not impose the restriction $m \leq r$ on the number of views.

${ }^{4}$ The sign is defined by $\prod_{i=1}^{m} \operatorname{sign}\left(I_{i}\right)$ where $\operatorname{sign}\left(I_{i}\right)$ is +1 or -1 depending on whether the sequence $\left(\operatorname{sort}\left(I_{i}\right) \operatorname{sort}\left(\bar{I}_{i}\right)\right)$ is an even or odd permutation, where $\bar{I}_{i}=\left\{1, \ldots, s_{i}\right\} \backslash I_{i}$ (see [3]).
}

where $\mathrm{U}_{i}^{\bar{I}_{i}}$ is the square submatrix of $\mathrm{U}_{i}$ made by choosing rows in $\bar{I}_{i}=\left\{1, \ldots, \alpha_{i}\right\} \backslash I_{i}$. Notice that $\operatorname{det}\left(\mathrm{U}_{i}^{\bar{I}_{i}}\right)$ for different values of $\bar{I}_{i}$ form the Grassmann coordinates of $\mathcal{C}\left(\mathrm{U}_{i}\right)$, the column space of $\mathrm{U}_{i}$. The main theorem of [3] states that the projection matrices $\mathrm{P}_{i}$ can be uniquely constructed from the Grassmann tensor, up to projectivity:

Theorem 1 ([3]). Consider a set of $m$ generic projection matrices $\mathrm{P}_{1}, \mathrm{P}_{2}, \ldots, \mathrm{P}_{m}$, with $\mathrm{P}_{i} \in \mathbb{R}^{s_{i} \times r}$, such that $m \leq r \leq \sum_{i=1}^{m}\left(s_{i}-1\right)$, and a valid profile $\left(\alpha_{1}, \alpha_{2}, \ldots, \alpha_{m}\right)$ for which $\alpha_{i} \geq 1$ for all $i$. Then if at least one $s_{i}$ is greater than 2 , the matrices $\mathrm{P}_{i}$ are determined up to a projective ambiguity from the set of minors of $\mathrm{P}$ chosen with $\alpha_{i}$ rows from each $\mathrm{P}_{i}$. If $s_{i}=2$ for all $i$, there are two equivalence classes of solutions.

The constructive proof of [3] gives a procedure to obtain the projections $\mathrm{P}_{i}$ from the Grassmann tensor. From each set of image point correspondences $\mathbf{x}_{1 j}, \mathbf{x}_{2 j}, \ldots, \mathbf{x}_{m j}$ one or more sets of subspaces $U_{1}, U_{2}, \ldots, U_{m}$ can be passed such that $\mathbf{x}_{i j} \in U_{i}$. Each choice of subspaces $U_{1}, \ldots, U_{m}$ gives a linear equation (6) on the elements of the tensor. The Grassmann tensor can be obtained as the null vector ${ }^{5}$ of the matrix of coefficients of the set of linear equations.

Lemma 3 ([7]). Consider a set of projection matrices $\mathrm{P}_{1}, \ldots, \mathrm{P}_{m}$ with $\mathrm{P}_{i} \neq 0$ for all $i$. Assume that there exists a valid profile $\boldsymbol{\alpha}=\left(\alpha_{1}, \alpha_{2}, \ldots, \alpha_{m}\right)$ with $\alpha_{k}=0$ such that $\mathcal{G}_{\boldsymbol{\alpha}}\left(\mathrm{P}_{1}, \ldots, \mathrm{P}_{m}\right)$ is nonzero. Then there exists another valid profile $\boldsymbol{\alpha}^{\prime}=\left(\alpha_{1}^{\prime}, \alpha_{2}^{\prime}, \ldots, \alpha_{m}^{\prime}\right)$ with $\alpha_{k}^{\prime}>0$ such that $\mathcal{G}_{\boldsymbol{\alpha}^{\prime}}\left(\mathrm{P}_{1}, \ldots, \mathrm{P}_{m}\right)$ is nonzero.

\section{The Grassmann tensor is unique}

This section asserts that if two configurations $\left(\left\{\mathrm{P}_{i}\right\},\left\{\mathbf{X}_{j}\right\}\right)$ and $\left(\left\{\hat{\mathrm{P}}_{i}\right\},\left\{\hat{\mathbf{X}}_{j}\right\}\right)$ project into the same image points $\mathbf{X}_{i j}$, then the associated Grassmann tensors of $\left\{\mathrm{P}_{i}\right\}$ and $\left\{\hat{\mathrm{P}}_{i}\right\}$ can only differ by a scaling factor. In other words, the Grassmann tensor is unique up to scale given a set of image points $\mathbf{x}_{i j}=\mathrm{P}_{i} \mathbf{X}_{j} / \lambda_{i j}$.

Theorem 2. Consider a setup $\left(\left\{\mathrm{P}_{i}\right\},\left\{\mathbf{X}_{j}\right\}\right)$ of $m$ generic projection matrices and $n$ points in general position and sufficiently many, and a valid profile $\boldsymbol{\alpha}=$ $\left(\alpha_{1}, \alpha_{2}, \ldots, \alpha_{m}\right)$ such that $\alpha_{i} \geq 1$ for all $i$. Now, for any other configuration $\left(\left\{\hat{\mathrm{P}}_{i}\right\},\left\{\hat{\mathbf{X}}_{j}\right\}\right)$ with $\hat{\mathbf{X}}_{j} \neq \mathbf{0}$ for all $j$, the set of relations

$$
\hat{\mathrm{P}}_{i} \hat{\mathbf{X}}_{j}=\hat{\lambda}_{i j} \mathrm{P}_{i} \mathbf{X}_{j}
$$

implies $\mathcal{G}_{\boldsymbol{\alpha}}\left(\hat{\mathrm{P}}_{1}, \ldots, \hat{\mathrm{P}}_{m}\right)=\beta \mathcal{G}_{\boldsymbol{\alpha}}\left(\mathrm{P}_{1}, \ldots, \mathrm{P}_{m}\right)$ for some scalar $\beta$.

\footnotetext{
${ }^{5}$ In Sect. 3 we prove that the Grassmann tensor is unique, meaning that the matrix of coefficients has a $1 \mathrm{D}$ null space.
} 
Notice that $\hat{\lambda}_{i j \text {-s }}$ have not been assumed to be nonzero. Considering the definition of a valid profile, the condition $\alpha_{i} \geq 1$ implies that the above theorem only applies when $m \leq r$. Here, we present an outline of the proof and refer the reader to [7] for the full proof.

We consider two submatrices $Q$ and $Q^{\prime}$ of $P=$ $\operatorname{stack}\left(\mathrm{P}_{1}, \ldots, \mathrm{P}_{m}\right)$ chosen according to the valid profile $\boldsymbol{\alpha}=\left(\alpha_{1}, \ldots, \alpha_{m}\right)$, such that all rows of $\mathbf{Q}$ and $\mathbf{Q}^{\prime}$ are equal except for the $l$-th rows $\mathbf{q}_{l}^{T}$ and $\mathbf{q}_{l}^{\prime T}$, which are chosen from different rows of $\mathrm{P}_{k}$ for some view $k$. We also represent by $\hat{Q}$ and $\hat{Q}^{\prime}$ the corresponding submatrices of $\hat{\mathrm{P}}=\operatorname{stack}\left(\hat{\mathrm{P}}_{1}, \ldots, \hat{\mathrm{P}}_{m}\right)$. Then we show that if $\operatorname{det}(\mathbf{Q}) \neq 0$, the equations (7) imply

$$
\operatorname{det}\left(\hat{\mathbf{Q}}^{\prime}\right)=\frac{\operatorname{det}\left(\mathbf{Q}^{\prime}\right)}{\operatorname{det}(\mathbf{Q})} \operatorname{det}(\hat{\mathbf{Q}}) .
$$

The rest of the proof is trivial: By starting with a submatrix Q of P according to $\boldsymbol{\alpha}$, and iteratively updating $Q$ by changing one row at a time in the way described above, we can finally traverse all possible submatrices chosen according to $\boldsymbol{\alpha}$. Due to genericity we assume that all submatrices of $\mathrm{P}$ chosen according to $\boldsymbol{\alpha}$ are non-singular ${ }^{6}$. Therefore, (8) implies that during the traversal the ratio $\beta=\operatorname{det}(\hat{\mathbf{Q}}) / \operatorname{det}(\mathbf{Q})$ stays the same. This means that each element of $\mathcal{G}_{\boldsymbol{\alpha}}\left(\hat{\mathrm{P}}_{1}, \ldots, \hat{\mathrm{P}}_{m}\right)$ is $\beta$ times the corresponding element of $\mathcal{G}_{\boldsymbol{\alpha}}\left(\mathrm{P}_{1}, \ldots, \mathrm{P}_{m}\right)$, implying $\mathcal{G}_{\boldsymbol{\alpha}}\left(\hat{\mathrm{P}}_{1}, \ldots, \hat{\mathrm{P}}_{m}\right)=\beta \mathcal{G}_{\boldsymbol{\alpha}}\left(\mathrm{P}_{1}, \ldots, \mathrm{P}_{m}\right)$.

The relation (8) is obtained in two steps. The first step is to write (7) in matrix form as

$$
\mathcal{M}\left(\mathbf{X}_{j}\right)\left(\begin{array}{c}
\hat{\boldsymbol{\lambda}}_{j} \\
\hat{\mathbf{X}}_{j}
\end{array}\right)=\mathbf{0}, \quad j=1,2, \ldots, n,
$$

where $\hat{\boldsymbol{\lambda}}_{j}=\left[\hat{\lambda}_{1 j}, \ldots, \hat{\lambda}_{m j}\right]^{T}$, and

$$
\mathcal{M}(\mathbf{X})=\left[\begin{array}{ccccc}
\mathrm{P}_{1} \mathbf{X} & & & & \hat{\mathrm{P}}_{1} \\
& \mathrm{P}_{2} \mathbf{X} & & & \hat{\mathrm{P}}_{2} \\
& & \ddots & & \vdots \\
& & & \mathrm{P}_{m} \mathbf{X} & \hat{\mathrm{P}}_{m}
\end{array}\right] .
$$

The matrix $\mathcal{M}(\mathbf{X})$ is $\left(\sum_{i} s_{i}\right) \times(m+r)$, and therefore a tall (or square) matrix. Due to the assumption $\hat{\mathbf{X}}_{j} \neq \mathbf{0}$ in Theorem 2, we conclude that $\mathcal{M}\left(\mathbf{X}_{j}\right)$ is rank deficient for all $\mathbf{X}_{j}$. This means that for any $(m+r) \times(m+r)$ submatrix $\mathcal{M}^{\prime}\left(\mathbf{X}_{j}\right)$ of $\mathcal{M}\left(\mathbf{X}_{j}\right)$ we have $\operatorname{det}\left(\mathcal{M}^{\prime}\left(\mathbf{X}_{j}\right)\right)=0$. As $\operatorname{det}\left(\mathcal{M}^{\prime}(\mathbf{X})\right)$ is a polynomial expression in $\mathbf{X}$, having $\operatorname{det}\left(\mathcal{M}^{\prime}\left(\mathbf{X}_{j}\right)\right)=0$ for sufficiently many points $\mathbf{X}_{j}$ in general position implies that $\operatorname{det}\left(\mathcal{M}^{\prime}(\mathbf{X})\right)$ is identically zero. The only other possibility is that $\mathbf{X}_{j}$-s all lie on a polynomial surface which can be ruled out by genericity.

\footnotetext{
${ }^{6}$ though the proof is possible under a milder assumption,
}

The second step is to choose a proper value for $\mathbf{X}$ and a proper submatrix $\mathcal{M}^{\prime}(\mathbf{X})$ of $\mathcal{M}(\mathbf{X})$, such that (8) follows from $\operatorname{det}\left(\mathcal{M}^{\prime}(\mathbf{X})\right)=0$. This proper choice for $\mathbf{X}$ is $\mathbf{Q}^{-1} \mathbf{e}_{l}$, where $\mathbf{e}_{l}$ is the $l$-th standard basis and $l$ is the row which is different in $\mathbf{Q}$ and $\mathbf{Q}^{\prime}$, as defined earlier. The submatrix $\mathcal{M}^{\prime}(\mathbf{X})$, is made by choosing the corresponding rows of $\mathrm{P}=\operatorname{stack}\left(\mathrm{P}_{1}, \ldots, \mathrm{P}_{m}\right)$ contributing to making $\mathrm{Q}$, choosing the corresponding row $\mathbf{q}_{l}^{\prime T}$ of $\mathbf{P}_{k}$ contributing to making $\mathbf{Q}^{\prime}$, and choosing one extra row from each $\mathrm{P}_{i}$ for $i \neq k$. See [7] for details.

\section{Projective Reconstruction}

Here, we state a theorem of projective reconstruction showing the projective equivalence of two setups $\left(\left\{\mathrm{P}_{i}\right\},\left\{\mathbf{X}_{j}\right\}\right)$ and $\left(\left\{\hat{\mathrm{P}}_{i}\right\},\left\{\hat{\mathbf{X}}_{j}\right\}\right)$ projecting into the same image points, given conditions on $\left(\left\{\hat{\mathrm{P}}_{i}\right\},\left\{\hat{\mathbf{X}}_{j}\right\}\right)$. Based on this, we present an alternative theorem in the next section with conditions on the projective depths $\hat{\lambda}_{i j}$.

Theorem 3 (Projective Reconstruction). Consider a setup of $m$ projections and $n$ points $\left(\left\{\mathrm{P}_{i}\right\},\left\{\mathbf{X}_{j}\right\}\right)$ where the matrices $\mathrm{P}_{i} \in \mathbb{R}^{s_{i} \times r}$ are generic, $\sum_{i=1}^{m}\left(s_{i}-1\right) \geq r$, and $s_{i} \geq 3$ for all views ${ }^{7}$, and the points $\mathbf{X}_{j} \in \mathbb{R}^{r}$ are sufficiently many and in general position. Given a second setup $\left(\left\{\hat{\mathrm{P}}_{i}\right\},\left\{\hat{\mathbf{X}}_{j}\right\}\right)$ which for all $i, j$ satisfies

$$
\hat{\mathrm{P}}_{i} \hat{\mathbf{X}}_{j}=\hat{\lambda}_{i j} \mathrm{P}_{i} \mathbf{X}_{j}
$$

for some scalars $\hat{\lambda}_{i j}$, if

(C1) $\hat{\mathrm{P}}_{i} \neq 0$ for all $i$, and

(C2) $\hat{\mathbf{X}}_{j} \neq \mathbf{0}$ for all $j$, and

(C3) there exists a non-singular $r \times r$ submatrix $\hat{\mathrm{Q}}$ of $\hat{\mathrm{P}}=\operatorname{stack}\left(\hat{\mathrm{P}}_{1}, \hat{\mathrm{P}}_{2}, \ldots, \hat{\mathrm{P}}_{m}\right)$ containing strictly fewer than $s_{i}$ rows from each $\hat{\mathrm{P}}_{i}$. (equivalently $\mathcal{G}_{\boldsymbol{\alpha}}\left(\hat{\mathrm{P}}_{1}, \ldots, \hat{\mathrm{P}}_{m}\right) \neq \mathbf{0}$ for some valid profile $\left.\boldsymbol{\alpha}\right)$,

then the two configurations $\left(\left\{\mathrm{P}_{i}\right\},\left\{\mathbf{X}_{j}\right\}\right)$ and $\left(\left\{\hat{\mathrm{P}}_{i}\right\},\left\{\hat{\mathbf{X}}_{j}\right\}\right)$ are projectively equivalent.

It is important to observe the theorem does not assume a priori that the projective depths $\hat{\lambda}_{i j}$ are nonzero. The outline of the proof is as follows. Given the profile $\boldsymbol{\alpha}=\left(\alpha_{1}, \ldots, \alpha_{m}\right)$ from condition (C3),

1. for the special case of $\alpha_{i} \geq 1$ for all $i$, using Theorem 2 , we have $\mathcal{G}_{\boldsymbol{\alpha}}\left(\hat{\mathrm{P}}_{1}, \ldots, \hat{\mathrm{P}}_{m}\right)=$ $\beta \mathcal{G}_{\boldsymbol{\alpha}}\left(\mathrm{P}_{1}, \ldots, \mathrm{P}_{m}\right)$, where $\beta$ is nonzero due to (C3).

2. As $\mathcal{G}_{\boldsymbol{\alpha}}\left(\hat{\mathrm{P}}_{1}, \ldots, \hat{\mathrm{P}}_{m}\right)$ is equal to $\mathcal{G}_{\boldsymbol{\alpha}}\left(\mathrm{P}_{1}, \ldots, \mathrm{P}_{m}\right)$ up to a nonzero scale, Theorem 1 by Hartley and

\footnotetext{
${ }^{7}$ We could have assumed the milder condition of $s_{i} \geq 3$ for at least one $i$. Our assumption avoids unnecessary complications.
} 
Schaffalitzky [3] suggests that $\left\{\mathrm{P}_{i}\right\}$ and $\left\{\hat{\mathrm{P}}_{i}\right\}$ are projectively equivalent. Using the Triangulation Lemma 2 we can then obtain that $\left(\left\{\mathrm{P}_{i}\right\},\left\{\mathbf{X}_{j}\right\}\right)$ and $\left(\left\{\hat{\mathrm{P}}_{i}\right\},\left\{\hat{\mathbf{X}}_{j}\right\}\right)$ are projectively equivalent.

3. To prove the theorem for the general case where some $\alpha_{i}$-s might be zero (and hence the number of views can be arbitrarily large), first notice that, by Lemma 3, different valid profiles $\boldsymbol{\alpha}^{\prime}$ can be chosen with a nonzero Grassmann tensor. Theorem 3 can be proven for the subset of views $i$ with $\alpha_{i}^{\prime} \neq 0$. The collection of such subsets due to different choices of $\boldsymbol{\alpha}^{\prime}$ span the whole set of views. Using Lemma 1 we can then stitch the projective equivalence for different subsets of views together and prove that for the whole set of views $\left(\left\{\mathrm{P}_{i}\right\},\left\{\mathbf{X}_{j}\right\}\right)$ and $\left(\left\{\hat{\mathbf{P}}_{i}\right\},\left\{\hat{\mathbf{X}}_{j}\right\}\right)$ are projectively equivalent.

\section{Restricting projective depths}

This section provides a second version of Theorem 3 in which it is assumed that $\hat{\lambda}_{i j}$-s are all nonzero, instead of putting restrictions on $\left(\left\{\hat{\mathrm{P}}_{i}\right\},\left\{\hat{\mathbf{X}}_{j}\right\}\right)$.

Theorem 4 (Projective Reconstruction). Consider a setup of $m$ projections and $n$ points $\left(\left\{\mathrm{P}_{i}\right\},\left\{\mathbf{X}_{j}\right\}\right)$ with $\mathrm{P}_{i} \in \mathbb{R}^{s_{i} \times r}$ generic, $s_{i} \geq 3$ for all views $i, \sum_{i=1}^{m}\left(s_{i}-\right.$ 1) $\geq r$, and the points $\mathbf{X}_{j} \in \mathbb{R}^{r}$ sufficiently many and in general position. Now, for any second configuration $\left(\left\{\hat{\mathrm{P}}_{i}\right\},\left\{\hat{\mathbf{X}}_{j}\right\}\right)$ satisfying

$$
\hat{\mathrm{P}}_{i} \hat{\mathbf{X}}_{j}=\hat{\lambda}_{i j} \mathrm{P}_{i} \mathbf{X}_{j} .
$$

for some nonzero scalars $\hat{\lambda}_{i j} \neq 0$, the configuration $\left(\left\{\hat{\mathrm{P}}_{i}\right\},\left\{\hat{\mathbf{X}}_{j}\right\}\right)$ is projectively equivalent to $\left(\left\{\mathrm{P}_{i}\right\},\left\{\mathbf{X}_{j}\right\}\right)$.

In Sect. 6 we will discuss that the theorem can be proved under milder restrictions than $\hat{\lambda}_{i j} \neq 0$ for all $i, j$. However, by proving projective equivalence, it eventually follows that $\hat{\lambda}_{i j}$-s are all nonzero. The next lemma is the key for the proof of the above theorem.

Lemma 4. Consider $m$ projection matrices $\hat{\mathrm{P}}_{1}, \hat{\mathrm{P}}_{2}, \ldots, \hat{\mathrm{P}}_{m}$ with $\hat{\mathrm{P}}_{i} \in \mathbb{R}^{s_{i} \times r}$, such that $\sum_{i=1}^{m}\left(s_{i}-1\right) \geq r$, and $\hat{\mathrm{P}}=\operatorname{stack}\left(\hat{\mathrm{P}}_{1}, \ldots, \hat{\mathrm{P}}_{m}\right)$ has full column rank $r$. If $\hat{\mathrm{P}}$ has no full-rank $r \times r$ submatrix created by choosing strictly fewer than $s_{i}$ rows from each $\hat{\mathrm{P}}_{i}$, then there exists a partition $\{I, J, K\}$ of the set of views $\{1,2, \ldots, m\}$, with $I \neq \emptyset$ (nonempty) and $\sum_{i \in I} s_{i}+\sum_{i \in J}\left(s_{i}-1\right) \leq r$, such that $\hat{\mathrm{P}}^{K}=\operatorname{stack}\left(\left\{\hat{\mathrm{P}}_{i}\right\}_{i \in K}\right)$ has rank $r^{\prime}=r-\sum_{i \in I} s_{i}-\sum_{i \in J}\left(s_{i}-1\right)$. Further, the row space of $\hat{\mathrm{P}}^{K}$ is spanned by the rows of an $r^{\prime} \times r$ submatrix $\hat{\mathrm{Q}}^{K}=\operatorname{stack}\left(\left\{\hat{\mathrm{Q}}_{i}\right\}_{i \in K}\right)$ of $\hat{\mathrm{P}}^{K}$, where each $\hat{\mathrm{Q}}_{i}$ is made by choosing strictly less than $s_{i}$ rows from $\hat{\mathrm{P}}_{i}$.
The proof of this lemma is quite long and intricate. The interested reader is referred to [7].

To prove Theorem 4 we just need to show that the condition $\hat{\lambda}_{i j} \neq 0$ implies (C1-C3) in Theorem 3. Assume that $\hat{\lambda}_{i j} \neq 0$ for some $i$ and $j$. Then from the genericity of $\mathrm{P}_{i}$ and $\mathbf{X}_{j}$ we have $\mathrm{P}_{i} \mathbf{X}_{j} \neq 0$, and thus $\hat{\mathrm{P}}_{i} \hat{\mathbf{X}}_{j}=\hat{\lambda}_{i j} \mathrm{P}_{i} \mathbf{X}_{j} \neq 0$, implying $\hat{\mathrm{P}}_{i} \neq 0$ and $\hat{\mathbf{X}}_{j} \neq \mathbf{0}$. Thus, $\hat{\lambda}_{i j} \neq 0$ for all $i, j$ implies (C1) and (C2).

The hardest part is to show that $\hat{\lambda}_{i j} \neq 0$ implies (C3), that is $\hat{\mathrm{P}}$ has a full-rank $r \times r$ submatrix chosen with strictly fewer than $s_{i}$ rows from each $\hat{\mathrm{P}}_{i}$. To get a contradiction, assume $\hat{\mathrm{P}}$ does not have such a submatrix. For the present, suppose that $\hat{P}$ has full column rank. Then by Lemma 4 , there exists a partition $\{I, J, K\}$ of views with $I \neq \emptyset$ and $\sum_{i \in I} s_{i}+$ $\sum_{i \in J}\left(s_{i}-1\right) \leq r$, such that $\hat{\mathrm{P}}^{K}=\operatorname{stack}\left(\left\{\hat{\mathrm{P}}_{i}\right\}_{i \in K}\right)$ has a row space of dimension $r^{\prime}=r-\sum_{i \in I} s_{i}-$ $\sum_{i \in J}\left(s_{i}-1\right)$, spanned by the rows of an $r^{\prime} \times r$ matrix $\hat{\mathbf{Q}}^{K}=\operatorname{stack}\left(\left\{\hat{\mathbf{Q}}_{i}\right\}_{i \in K}\right)$, where each $\hat{\mathrm{Q}}_{i}$ consists of strictly less than $s_{i}$ rows $^{8}$ from $\hat{\mathrm{P}}_{i}$. By relabeling the views if necessary, we assume that $\hat{\mathrm{P}}^{K}=\operatorname{stack}\left(\hat{\mathrm{P}}_{1}, \ldots, \hat{\mathrm{P}}_{l}\right)$ and $\hat{\mathrm{Q}}^{K}=\operatorname{stack}\left(\hat{\mathrm{Q}}_{1}, \ldots, \hat{\mathrm{Q}}_{l}\right)$. As rows of $\hat{\mathrm{Q}}^{K}$ span the row space of $\hat{\mathrm{P}}^{K}$, we have $\hat{\mathrm{P}}^{K}=\mathrm{A} \hat{\mathrm{Q}}^{K}$ for some $\left(\sum_{i=1}^{l} s_{i}\right) \times r^{\prime}$ matrix A. From (12), we have $\hat{\mathbf{P}}_{i} \hat{\mathbf{X}}_{j}=\hat{\lambda}_{i j} \mathrm{P}_{i} \mathbf{X}_{j}$ and, as a result, $\hat{\mathbf{Q}}_{i} \hat{\mathbf{X}}_{j}=\hat{\lambda}_{i j} \mathbf{Q}_{i} \mathbf{X}_{j}$, where $\mathbf{Q}_{i}$ is the submatrix of $\mathrm{P}_{i}$ corresponding to $\hat{\mathbf{Q}}_{i}$. This gives

$$
\begin{aligned}
\hat{\mathrm{P}}^{K} \hat{\mathbf{X}}_{j} & =\operatorname{diag}\left(\mathrm{P}_{1} \mathbf{X}_{j}, \mathrm{P}_{2} \mathbf{X}_{j}, \ldots, \mathrm{P}_{l} \mathbf{X}_{j}\right) \hat{\boldsymbol{\lambda}}_{j} \\
\hat{\mathrm{Q}}^{K} \hat{\mathbf{X}}_{j} & =\operatorname{diag}\left(\mathrm{Q}_{1} \mathbf{X}_{j}, \mathrm{Q}_{2} \mathbf{X}_{j}, \ldots, \mathrm{Q}_{l} \mathbf{X}_{j}\right) \hat{\boldsymbol{\lambda}}_{j}
\end{aligned}
$$

where $\operatorname{diag}(\cdot)$ is the block diagonal matrix of its arguments, and $\hat{\boldsymbol{\lambda}}_{j}=\left[\hat{\lambda}_{1 j}, \ldots, \hat{\lambda}_{l j}\right]^{T}$. From $\hat{\mathrm{P}}^{K}=\mathrm{A} \hat{\mathrm{Q}}^{K}$ we get

$$
\mathcal{M}\left(\mathbf{X}_{j}\right) \hat{\boldsymbol{\lambda}}_{j}=\mathbf{0}
$$

where

$$
\begin{aligned}
\mathcal{M}(\mathbf{X})= & \operatorname{diag}\left(\mathrm{P}_{1} \mathbf{X}, \mathrm{P}_{2} \mathbf{X}, \ldots, \mathrm{P}_{l} \mathbf{X}\right) \\
& -\mathrm{A} \operatorname{diag}\left(\mathbf{Q}_{1} \mathbf{X}, \mathbf{Q}_{2} \mathbf{X}, \ldots, \mathrm{Q}_{l} \mathbf{X}\right) .
\end{aligned}
$$

Notice that, $\mathcal{M}(\mathbf{X})$ is $\left(\sum_{i=1}^{l} s_{i}\right) \times l$, and thus a tall matrix. As $\hat{\boldsymbol{\lambda}}_{j} \neq \mathbf{0}$ (since $\hat{\lambda}_{i j} \neq 0$ for all $i, j$ ), $\mathcal{M}\left(\mathbf{X}_{j}\right) \hat{\boldsymbol{\lambda}}_{j}=$ $\mathbf{0}$ implies that $\mathcal{M}\left(\mathbf{X}_{j}\right)$ is rank deficient, and thus, all of its $l \times l$ submatrices have a zero determinant. Since for any $l \times l$ submatrix $\mathcal{M}^{\prime}(\mathbf{X})$ of $\mathcal{M}(\mathbf{X})$ the expression $\operatorname{det}\left(\mathcal{M}^{\prime}(\mathbf{X})\right)$ is a polynomial on $\mathbf{X}$ which is equal to zero at sufficiently many points $\mathbf{X}_{j}$ in general position, we can say that $\operatorname{det}\left(\mathcal{M}^{\prime}(\mathbf{X})\right)$ is identically zero ${ }^{9}$ for all submatrices $\mathcal{M}^{\prime}(\mathbf{X})$ of $\mathcal{M}(\mathbf{X})$. It follows that $\mathcal{M}(\mathbf{X})$ is

\footnotetext{
${ }^{8}$ It might happen that for some $i \in K$ no row of $\hat{\mathrm{P}}_{i}$ is contained in $\hat{Q}^{K}$. In this case one can think of $\hat{\mathbf{Q}}_{i}$ as a matrix with zero rows.

${ }^{9}$ otherwise all $\mathbf{X}_{j}$-s must lie on a polynomial surface, which can be ruled out by genericity.
} 
rank deficient for all $\mathbf{X}$. As $\hat{\mathbf{Q}}^{K}$ is $r^{\prime} \times r$ with $r^{\prime}<r$ and $\mathrm{P}_{i}$-s are generic, we can take a nonzero vector $\mathbf{Y}$ in the null space of $\hat{\mathrm{Q}}^{K}$ such that no matrix $\hat{\mathrm{P}}_{i}$ for $i=1, \ldots, l$ has $\mathbf{Y}$ in its null space ${ }^{10}$. In this case, we have $\mathbf{Q}_{i} \mathbf{Y}=0$ for all $i$, implying $\mathcal{M}(\mathbf{Y})=\operatorname{diag}\left(\mathrm{P}_{1} \mathbf{Y}, \ldots, \mathrm{P}_{l} \mathbf{Y}\right)$. Now, from $\mathbf{Y} \notin \mathcal{N}\left(\hat{\mathrm{P}}_{i}\right)$, we have $\mathrm{P}_{i} \mathbf{Y} \neq 0$ for $i=1, \ldots, l$. This implies that $\mathcal{M}(\mathbf{Y})=\operatorname{diag}\left(\mathrm{P}_{1} \mathbf{Y}, \ldots, \mathrm{P}_{l} \mathbf{Y}\right)$ has full column rank, contradicting the fact that $\mathcal{M}(\mathbf{X})$ is rank deficient for all $\mathcal{M}(\mathbf{X})$. Therefore, condition (C3) in Theorem 3 also holds, which completes the proof of Theorem 4 for when $\hat{P}$ has full column rank. One can show that this is always the case and the rank deficiency assumption about $\hat{\mathrm{P}}$ leads to contradiction. The trick is to consider the matrix $\hat{\mathbf{X}}=\left[\hat{\mathbf{X}}_{1}, \ldots, \hat{\mathbf{X}}_{m}\right]$, refactorize $\hat{\mathrm{P}} \hat{\mathrm{X}}$ as $\hat{\mathrm{P}}^{\prime} \hat{\mathrm{X}}^{\prime}$ such that $\hat{\mathrm{P}}^{\prime}$ has full column rank, prove projective equivalence for $\left(\left\{\hat{\mathrm{P}}_{i}^{\prime}\right\},\left\{\hat{\mathbf{X}}_{j}^{\prime}\right\}\right)$, and finally, show that $\hat{\mathrm{P}}$ has full column rank from $\hat{\mathrm{P}} \hat{\mathrm{X}}=\hat{\mathrm{P}}^{\prime} \hat{\mathrm{X}}^{\prime}$. The reader can refer to [7] for a more detailed proof.

\section{Wrong solutions to projective factor- ization}

Let us write equations $\hat{\lambda}_{i j} \mathbf{x}_{i j}=\hat{\mathrm{P}}_{i} \hat{\mathbf{X}}_{j}$ in matrix form

$$
\hat{\Lambda} \odot\left[\mathbf{x}_{i j}\right]=\hat{\mathrm{P}} \hat{\mathrm{X}},
$$

where $\hat{\Lambda}=\left[\hat{\lambda}_{i j}\right], \quad \hat{\Lambda} \odot\left[\mathbf{x}_{i j}\right]=\left[\hat{\lambda}_{i j} \mathbf{x}_{i j}\right], \quad \hat{\mathrm{P}}=$ $\operatorname{stack}\left(\hat{\mathrm{P}}_{1}, \ldots, \hat{\mathrm{P}}_{m}\right)$ and $\hat{\mathrm{X}}=\left[\hat{\mathbf{X}}_{1}, \ldots, \hat{\mathbf{X}}_{n}\right]$. The projective factorization algorithms seek to find $\hat{\Lambda}$ such that $\hat{\Lambda} \odot\left[\mathbf{x}_{i j}\right]$ can be factorized as the product of a $\left(\sum_{i} s_{i}\right) \times r$ matrix $\hat{\mathrm{P}}$ by an $r \times n$ matrix $\hat{\mathrm{X}}$. If $\mathbf{x}_{i j}$-s are obtained from $\left(\left\{\mathrm{P}_{i}\right\},\left\{\mathbf{X}_{j}\right\}\right)$ as $\mathbf{x}_{i j}=\mathrm{P}_{i} \mathbf{X}_{j} / \lambda_{i j}$, our theory says that any solution $(\hat{\Lambda}, \hat{\mathrm{P}}, \hat{\mathrm{X}})$ to $(17)$ is equivalent to the true solution $(\Lambda, P, X)$, provided that $(\hat{\Lambda}, \hat{P}, \hat{\mathrm{X}})$ satisfies certain restrictions, such as conditions (C1-C3) on $\hat{P}$ and $\hat{\mathrm{X}}$ in Theorem 3 , or $\hat{\Lambda}$ having no zero element in Theorem 4. It is worth to see what degenerate (projectively nonequivalent) forms a solution $(\hat{\Lambda}, \hat{\mathrm{P}}, \hat{\mathrm{X}})$ to $(17)$ can get when such restrictions are not completely imposed. This is important in the factorization-based methods, in which sometimes such restrictions cannot be efficiently implemented.

The reader can check that Theorem 4 can be proved under weaker assumptions than $\hat{\lambda}_{i j} \neq 0$, as follows

(D1) The matrix $\hat{\Lambda}=\left[\hat{\lambda}_{i j}\right]$ has no zero rows,

(D2) The matrix $\hat{\Lambda}=\left[\hat{\lambda}_{i j}\right]$ has no zero columns,

(D3) For every partition $\{I, J, K\}$ of views $\{1,2, \ldots, m\}$ with $I \neq \emptyset$ and $\sum_{i \in I} s_{i}+\sum_{j \in J}\left(s_{j}-1\right)<r$, the matrix $\hat{\Lambda}^{K}$ has sufficiently many nonzero columns,

\footnotetext{
${ }^{10} \mathbf{Y}$ must be chosen from $\mathcal{N}\left(\mathrm{Q}^{K}\right) \backslash \cup \cup_{i=1}^{l}\left(\mathcal{N}\left(\mathrm{Q}^{K}\right) \cap \mathcal{N}\left(\mathrm{P}_{i}\right)\right)$ which is nonempty (in fact open and dense in $\mathcal{N}\left(\mathrm{Q}^{K}\right)$ ) for generic $\mathrm{P}_{i}$-s.
}

where $\hat{\Lambda}^{K}$ is the submatrix of $\hat{\Lambda}$ created by selecting rows according to $K$.

Notice that (D1) and (D2) respectively guarantee (C1) and $(\mathrm{C} 2)$ in Theorem 3 due to $\mathrm{P}_{i} \mathbf{X}_{j} \neq 0$ (by genericity) and $\hat{\mathrm{P}}_{i} \hat{\mathbf{X}}_{j}=\hat{\lambda}_{i j} \mathrm{P}_{i} \mathbf{X}_{j}$. The condition (D3) guarantees that the vector $\hat{\boldsymbol{\lambda}}_{j}$ used in (15), which is the $j$-th column of $\hat{\Lambda}^{K}$ defined in (D3), is nonzero for sufficiently many $j$-s. Therefore, (D3) is used to guarantee (C3), that is $\hat{P}$ has a nonzero minor chosen according to some valid profile ${ }^{11}$. The discussion under (16) implies that "sufficiently many" in (D3) means as many such that $\mathcal{M}(\mathbf{X})$ is guaranteed to be rank deficient for all $\mathbf{X}$.

It is trivial to see how violating (D1) and (D2) can lead to a false solution to (17). For example set $\hat{\mathrm{X}}=\mathrm{X}$, $\hat{\mathrm{P}}_{k}$ and the $k$-th row of $\hat{\Lambda}$ equal to zero, and the rest of $\hat{\mathrm{P}}$ and $\hat{\Lambda}$ equal to $P$ and $\Lambda$. In what comes next, we assume that (D1) and (D2) hold, that is $\hat{\Lambda}$ has no zero rows or zero columns, and look for less trivial false solutions to (17). According to our discussion above, for this class of wrong solutions conditions (C3) about $\hat{P}$ and (D3) about $\hat{\Lambda}$ must be violated. This means that the set of views $\{1,2, \ldots, m\}$ can be partitioned into $I, J, K$ with $I$ nonempty and $\sum_{i \in I} s_{i}+\sum_{i \in J}\left(s_{i}-1\right)<r$, such that the submatrix $\hat{\Lambda}^{K}$ of $\hat{\Lambda}$ has few nonzero columns. Moreover, by Lemma 4, the submatrix $\hat{\mathrm{P}}^{K}$ of $\hat{\mathrm{P}}$ has rank $r^{\prime}=r-\sum_{i \in I} s_{i}-\sum_{i \in J}\left(s_{i}-1\right)$. Here, we show how this can happen with a simple example in which $J=\emptyset$. It is possible, however, to show that degenerate solutions exist for every partition $\{I, J, K\}$ satisfying the properties mentioned above.

For a setup $\left(\left\{\mathrm{P}_{i}\right\},\left\{\mathbf{X}_{j}\right\}\right)$, partition the views into $I$ and $K$, such that $\sum_{i \in I} s_{i}<r$. Split $\mathrm{P}$ row-wise into submatrices $\mathrm{P}^{I}$ and $\mathrm{P}^{K}$. By possibly relabeling the views, we assume that $\mathrm{P}=\operatorname{stack}\left(\mathrm{P}^{I}, \mathrm{P}^{K}\right)$. Notice that $\mathrm{P}^{I}$ has $\sum_{i \in I} s_{i}$ rows and $r$ columns, and therefore, at least an $r^{\prime}=r-\sum_{i \in I} s_{i}$ dimensional null space. Consider an $r \times r^{\prime}$ matrix N with orthonormal columns all in the null space of $\mathrm{P}^{I}$. Also, let $\mathrm{R}$ be the orthogonal projection matrix into the row space of $\mathrm{P}^{I}$. Divide the matrix $\mathbf{X}=\left[\mathbf{X}_{1}, \ldots, \mathbf{X}_{m}\right]$ into two parts as $\mathbf{X}=\left[\mathrm{X}_{1}, \mathbf{X}_{2}\right]$ where $\mathbf{X}_{1}=\left[\mathbf{X}_{1}, \ldots, \mathbf{X}_{r^{\prime}}\right]$ and $\mathbf{X}_{2}=\left[\mathbf{X}_{r^{\prime}+1}, \ldots, \mathbf{X}_{m}\right]$. Define the corresponding submatrices of $\hat{\mathrm{P}}$ and $\hat{\mathrm{X}}$ as

$$
\begin{array}{ll}
\hat{\mathrm{P}}^{I}=\mathrm{P}^{I}, & \hat{\mathrm{P}}^{K}=\mathrm{P}^{K} \mathrm{X}_{1} \mathrm{~N}^{T}, \\
\hat{\mathrm{X}}_{1}=\mathrm{RX}_{1}+\mathrm{N}, & \hat{\mathrm{X}}_{2}=\mathrm{RX}_{2} .
\end{array}
$$

One can easily check that

$$
\hat{\mathrm{P}} \hat{\mathrm{X}}=\left[\begin{array}{c}
\hat{\mathrm{P}}^{I} \\
\hat{\mathrm{P}}^{K}
\end{array}\right]\left[\hat{\mathrm{X}}_{1}, \hat{\mathrm{X}}_{2}\right]=\left[\begin{array}{cc}
\mathrm{P}^{I} \mathrm{X}_{1} & \mathrm{P}^{I} \mathrm{X}_{2} \\
\mathrm{P}^{K} \mathrm{X}_{1} & 0
\end{array}\right]=\hat{\Lambda} \odot(\mathrm{PX})
$$

\footnotetext{
${ }^{11}$ Notice that (D3) does not consider the case of $\sum_{i \in I} s_{i}+$ $\sum_{j \in J}\left(s_{j}-1\right)=r$. One can show that in this case $K$ must be non-empty and $\hat{\mathrm{P}}^{K}$ must be zero [7]. This is precluded by (D1).
} 
where $\hat{\Lambda}$ has a block structure of the form

$$
\hat{\Lambda}=\left[\begin{array}{c}
\hat{\Lambda}^{I} \\
\hat{\Lambda}^{K}
\end{array}\right]=\left[\begin{array}{ll}
1 & 1 \\
1 & 0
\end{array}\right]
$$

As $\hat{\mathrm{P}}^{I} \in \mathbb{R}^{\left(r-r^{\prime}\right) \times r}$ at most has rank $r-r^{\prime}$ and $\hat{\mathrm{P}}^{K}=$ $\mathrm{P}^{K} \mathrm{X}_{1} \mathrm{~N}^{T}$ (with $\mathrm{N} \in \mathbb{R}^{r \times r^{\prime}}$ ) at most has rank $r^{\prime}$, if $\hat{\mathrm{P}}=\operatorname{stack}\left(\hat{\mathrm{P}}^{I}, \hat{\mathrm{P}}^{K}\right)$ has maximal rank $r$ then $\hat{\mathrm{P}}^{K}$ has to have rank $r^{\prime}$, as also confirmed by Lemma 4. One can check that $\hat{\mathrm{P}}=\operatorname{stack}\left(\hat{\mathrm{P}}^{I}, \hat{\mathrm{P}}^{K}\right)$ has no full rank submatrix chosen by less than $s_{i}$ rows from each $\hat{\mathrm{P}}_{i}$. Thus, (C3) is violated and the Grassmann tensor of $\left\{\hat{\mathrm{P}}_{i}\right\}$ corresponding to any valid profile is zero. Also, observe that in (21) the submatrix $\hat{\Lambda}^{K}$ of $\hat{\Lambda} \in \mathbb{R}^{m \times n}$ has only $r^{\prime}$ nonzero columns, no matter how large $n$ is. This is how (D3) is violated.

Using the above style for finding wrong solutions, $\hat{\Lambda}^{K}$ can have at most $r^{\prime}$ nonzero columns. Unfortunately, this is not always the case. Instead, the limit for the number of nonzero columns allowable in a wrong solution is as many such that the rank of $\hat{\Lambda}^{K} \odot\left(\mathrm{P}^{K} \mathrm{X}\right)$ is not more than $r^{\prime}$. This is necessary for having a wrong solution as $\hat{\Lambda}^{K} \odot\left(\mathrm{P}^{K} \mathrm{X}\right)=\hat{\mathrm{P}}^{K} \hat{\mathrm{X}}$, and $\hat{\mathrm{P}}^{K}$ cannot have a rank of more than $r^{\prime}$ according to Lemma 4. One can also show that this is a sufficient condition for having a wrong solution. With a little more complication, one can as well construct examples of wrong solution for partitions $\{I, J, K\}$ with $J$ nonempty.

For the classic case of $\mathbb{P}^{3} \rightarrow \mathbb{P}^{2}$ projections $(r=$ $\left.4, s_{i}=3\right)$, the only possible partition $\{I, J, K\}$ is when $I$ is a singleton and $J$ is empty. This is due to the conditions $I \neq \emptyset$ and $\sum_{i \in I} s_{i}+\sum_{j \in J}\left(s_{j}-1\right)<r=4$. In this case, $\hat{\Lambda}^{I}$ consists of only one row. Further, we have $r^{\prime}=r-\sum_{i \in I} s_{i}-\sum_{j \in J}\left(s_{j}-1\right)=4-3-0=1$. The reader can check that the condition $\operatorname{Rank}\left(\hat{\Lambda}^{K} \odot\right.$ $\left.\left(\mathrm{P}^{K} \mathrm{X}\right)\right) \leq r^{\prime}=1$, implies that only one column of $\hat{\Lambda}^{K}$ can be nonzero, causing $\hat{\Lambda}$ to have a cross-shaped structure. Therefore, the theory given in the authors' previous work [8] follows as a special case.

\section{Conclusion}

This paper investigates projective reconstruction for arbitrary dimensional projections. We obtain the following results for a generic setup:

- The multi-view (Grassmann) tensor is unique, up to a scaling factor, given the image points $\mathbf{x}_{i j}$.

- Any solution to the set of equations $\hat{\lambda}_{i j} \mathbf{x}_{i j}=$ $\hat{\mathrm{P}}_{i} \hat{\mathbf{X}}_{j}$ is projectively equivalent to the true setup, if the $\hat{\mathrm{P}}_{i}$-S and $\hat{\mathbf{X}}_{j-\mathrm{S}}$ are nonzero and $\hat{\mathrm{P}}=$ $\operatorname{stack}\left(\hat{\mathrm{P}}_{1}, \ldots, \hat{\mathrm{P}}_{m}\right)$ has at least one non-singular $r \times r$ submatrix created by choosing strictly fewer than $s_{i}$ rows from each $\hat{\mathrm{P}}_{i} \in \mathbb{R}^{s_{i} \times r}$.
- Any solution to the set of equations $\hat{\lambda}_{i j} \mathbf{x}_{i j}=\hat{\mathrm{P}}_{i} \hat{\mathbf{X}}_{j}$ is projectively equivalent to the true setup if $\hat{\lambda}_{i j}$ is nonzero for all $i, j$.

- False solutions to the projective factorization problem $\hat{\Lambda} \odot\left[\mathbf{x}_{i j}\right]=\hat{\mathrm{P}} \hat{\mathrm{X}}$ in general case can be much more complex than in the case of projections $\mathbb{P}^{3} \rightarrow \mathbb{P}^{2}$, as demonstrated in Sect. 6 .

A possible extension to this work is to consider the case of incomplete data, where some image points $\mathbf{x}_{i j}$ are missing. It would be also useful to compile a simplified list of all the required generic properties needed for the proof of projective reconstruction. This is because in almost all applications the projection matrices and points have a special structure, meaning they are members of a nongeneric set. It is now a nontrivial question whether the restriction of the genericity conditions to this nongeneric set is relatively generic.

\section{References}

[1] Y. Dai, H. Li, and M. He. Projective multi-view structure and motion from element-wise factorization. PAMI, PP(99):1-1, 2013.

[2] R. Hartley and R. Vidal. Perspective nonrigid shape and motion recovery. pages $276-289,2008$.

[3] R. I. Hartley and F. Schaffalitzky. Reconstruction from projections using Grassmann tensors. In European Conference on Computer Vision, 2004.

[4] R. I. Hartley and A. Zisserman. Multiple View Geometry in Computer Vision. Cambridge University Press, second edition, 2004.

[5] A. Heyden. A common framework for multiple view tensors. In ECCV, pages 3-19, 1998.

[6] S. Mahamud, M. Hebert, Y. Omori, and J. Ponce. Provably-convergent iterative methods for projective structure from motion. In CVPR, pages 1018-1025, 2001.

[7] B. Nasihatkon. Generalizations of the Projective Reconstruction Theorem. $\mathrm{PhD}$ thesis, Australian National University, Canberra, Australia, 2014.

[8] B. Nasihatkon, R. Hartley, and J. Trumpf. A generalized projective reconstruction theorem. preprint, 2013.

[9] J. Oliensis and R. Hartley. Iterative extensions of the Sturm/Triggs algorithm: convergence and nonconvergence. PAMI, 29(12):2217 - 2233, 2007.

[10] P. F. Sturm and B. Triggs. A factorization based algorithm for multi-image projective structure and motion. In $E C C V$, pages 709-720, 1996.

[11] B. Triggs, P. F. McLauchlan, R. I. Hartley, and A. W. Fitzgibbon. Bundle adjustment - a modern synthesis. In ICCV Proceedings of the International Workshop on Vision Algorithms, pages 298-372, 2000.

[12] L. Wolf and A. Shashua. On projection matrices $\mathbb{P}^{k} \rightarrow$ $\mathbb{P}^{2}, k=3, \ldots, 6$, and their applications in computer vision. IJCV, 48(1):53-67, 2002. 